\title{
LETTER
}

\section{Flux dependence of carbon chemical erosion by deuterium ions}

\author{
J. Roth ${ }^{1}$, R. Preuss ${ }^{1}$, W. Bohmeyer ${ }^{2}$, S. Brezinsek ${ }^{3}$, A. Cambe ${ }^{4}$, \\ E. Casarotto ${ }^{3}$, R. Doerner ${ }^{5}$, E. Gauthier ${ }^{4}$, G. Federici ${ }^{6}$, \\ S. Higashijima ${ }^{7}$, J. Hogan $^{4}$, A. Kallenbach ${ }^{1}$, A. Kirschner ${ }^{3}$, \\ H. Kubo ${ }^{7}$, J.M. Layet ${ }^{4}$, T. Nakano ${ }^{7}$, V. Philipps ${ }^{3}$, A. Pospieszczyk ${ }^{3}$, \\ R. Pugno ${ }^{1}$, R. Ruggiéri ${ }^{4}$, B. Schweer ${ }^{3}$, G. Sergienko ${ }^{3}$ and \\ M. Stamp ${ }^{8}$ \\ ${ }^{1}$ Max-Planck-Institut für Plasmaphysik, EURATOM Association, D-85748 Garching, \\ Germany \\ ${ }^{2}$ Max-Planck-Institut für Plasmaphysik, EURATOM Association, Mohrenstr. 42, \\ D-10117 Berlin, Germany \\ ${ }^{3}$ Institut für Plasmaphysik, Forschungszentrum Jülich, EURATOM Association, \\ D-52425 Jülich, Germany \\ ${ }^{4}$ Association EURATOM-CEA, CEA Cadarache, F-13018 St Paul lez Durance, France \\ ${ }^{5}$ Fusion Energy Research Program, University of California San Diego, La Jolla, \\ CA 92093-0417, USA \\ ${ }^{6}$ ITER JWS Garching Co-center, Boltzmannstr. 2, D-85748 Garching, Germany \\ ${ }^{7}$ Japan Atomic Energy Research Institute, Naka-machi, Maka-gun, Ibaraki-ken 311-0193, \\ Japan \\ ${ }^{8}$ EURATOM/UKAEA Fusion Association, Culham Science Center, \\ Abingdon OX14 3DB, UK
}

Received 12 May 2004, accepted for publication 22 September 2004

Published 2 November 2004

Online at stacks.iop.org/NF/44/L21

doi:10.1088/0029-5515/44/11/L01

\begin{abstract}
The chemical erosion of carbon in interaction with a hydrogen plasma has been studied in detail in ion beam experiments, and erosion yield values are available as a function of ion energy and surface temperature. However, the conditions in the ITER divertor cannot be simulated by ion beam experiments, especially as far as ion flux is concerned.

Therefore, a joint attempt was made through the EU Task Force on plasma-wall interaction and the international tokamak physics activity involving seven different fusion devices and plasma simulators to clarify the flux dependence. For each data point the local plasma conditions were normalized to an impact energy of $30 \mathrm{eV}$, care was taken to select data for a surface temperature close to the maximum yield or room temperature and the calibration of the diagnostic was performed in situ. Through this procedure the previous large scatter was significantly reduced, revealing a clear trend for a decreasing yield with increasing ion flux, $\Phi$. After the attribution of an error to each data point a fit using Bayesian probability analysis was performed, yielding a decrease in the erosion yield with $\Phi^{-0.54}$ at high ion fluxes.
\end{abstract}

\section{Introduction}

The erosion of plasma-facing materials in magnetically confined fusion devices, such as ITER, is a key issue from several aspects: component lifetime and plasma contamination by eroded surface atoms are longstanding issues for material choice in ITER. Safe management and accounting of tritium in ITER and future fusion power reactors will be crucial for the public acceptance of fusion as an environmentally benign power source. The issue of tritium retention is dominated by the inventory retained in deposited layers of eroded material, so that again, erosion as the starting point of processes leading to the build-up of tritium inventory, is of prime importance.

Erosion due to energetic particle bombardment depends on a number of parameters such as the mass ratio of incident particles to surface atoms, particle energy and flux, as well as 
surface temperature. There has been a remarkable progress in our understanding of the fundamental reaction steps in chemical erosion over the last decade. However, there are still issues, related to the special conditions in fusion applications, e.g. erosion at high fluxes and low particle energies, which cannot be simulated in ion beam experiments, and therefore, need special attention. This paper presents data from ion beams, plasma simulators and erosion measurements in the plasma edge, covering four orders of magnitude in ion flux for detailed analysis of the possible flux dependence of chemical erosion.

\section{Present understanding of atomistic processes of chemical erosion}

Over the last decade considerable progress has been made in our fundamental understanding of chemical erosion of carbon [1]. The thorough investigation by Horn et al [2] on the thermal chemical reactivity has been extended to the case of energetic ion bombardment, by Roth and García-Rosales [3]. Simultaneously, new ion beam experiments at energies down to $10 \mathrm{eV} \mathrm{[4-6]} \mathrm{have} \mathrm{improved} \mathrm{the} \mathrm{understanding} \mathrm{of} \mathrm{ion-induced}$ hydrocarbon emission.

Three processes determine the chemical erosion of carbon under low-energy hydrogen bombardment [3]:

(1) The reaction of thermalized ions within the implanted surface proceeds via the hydrogenation of carbon atoms at the edges of graphitic planes to $\mathrm{CH}_{3}-\mathrm{C}$ complexes. At temperatures above $400 \mathrm{~K} \mathrm{CH}_{3}$ radicals can be released, while at temperatures above $600 \mathrm{~K}$ recombinative hydrogen release $\left(\mathrm{H}_{2}\right)$ starts to reduce the chemical erosion yield. This thermal chemical erosion was elucidated in detail by Küppers and co-workers [2,7], quantifying all reaction cross sections and activation energies. The complete temperature dependence was described analytically by Roth and García-Rosales [3]. For the thermal reaction, no dependence on the hydrogen isotope was observed.

(2) The thermal reaction is enhanced by radiation damage introduced in the material that provides open bonds for hydrogen attachment. Damage is created by kinetic energy transfer from incident ions to lattice atoms and is, therefore, responsible for the dependence of the chemical erosion yield on the hydrogen isotope. The basic thermal reaction below the threshold for damage production depends strongly on the crystalline perfection of the carbon material, with maximum erosion yields between $10^{-3}$ for well annealed pyrolytic graphite and $10^{-1}$ for amorphous a-C:D layers [8]. At energies where radiation damage amorphizes the graphite lattice, the strong dependence on the material structure disappears [9].

(3) At low surface temperatures all broken carbon bonds are essentially hydrated, but no thermal release of hydrocarbons occurs. However, hydrocarbon radicals are bound to the surface with much smaller binding energy $(\approx 1 \mathrm{eV})$ than are carbon surface atoms in their regular lattice environment $(7.4 \mathrm{eV})$. This leads to ioninduced desorption of hydrocarbon radicals, which can be described in a manner analogous to damage production using a threshold energy, $E_{\text {des }}$, in the low electronvolt range. This process does not only produce saturated hydrocarbons, but also hydrocarbon radicals, as has been demonstrated by collector experiments performed by Balden and Roth [6]. They showed that only 50\% of the emitted species can be detected as saturated $\mathrm{CH}_{4}$ molecules in residual gas analysis and about $50 \%$ of the eroded carbon atoms were retained on Al-collector strips in front of the target.

The clarification of the chemical reaction cycle including all cross sections and reaction rates allows the formulation of a set of analytical equations describing the erosion yield as a function of surface temperature, ion energy and ion flux $[3,10]$. The absolute erosion yield and the temperature, $T_{\max }$, where the yield reaches the maximum are well reproduced, although a slight shift in $T_{\max }$ to lower temperatures with decreasing energy is not included in the model. Like the temperature dependence, the energy dependence of chemical sputtering can also be reproduced adequately using an analytic formula [10].

\section{Flux dependence}

The investigation and description of these processes have been performed for thermal hydrogen atom or ion fluxes of the order of $10^{16}-10^{20} \mathrm{~m}^{-2} \mathrm{~s}^{-1}$. The thermal reaction cycle predicts a pronounced shift in the temperature, $T_{\max }$, where the maximum erosion yield occurs towards higher temperatures with increasing ion flux. This temperature shift with ion flux is well reproduced in the experimental data [11]. However, at fluxes above $10^{21} \mathrm{~m}^{-2} \mathrm{~s}^{-1}$ as reached in plasma simulators or under tokamak conditions, the maximum temperature, $T_{\max }$, reaches values of about $1000 \mathrm{~K}$. At these elevated temperatures, the thermodynamic equilibrium of the $\mathrm{H} / \mathrm{C}$ system shifts from $\mathrm{CH}_{4}$ formation to $\mathrm{H}_{2}$ release [12] and the erosion yield is expected to decrease with ion flux. Additionally, the onset of graphitization will lead to annealing of radiation damage, resulting in suppression of the reactivity of the carbon material.

This has led to the prediction that at such high fluxes the yield at $T_{\max }$ decreases. This was supported by the first highflux investigations on hydrocarbon production at the limiter in DITE [13], where hardly any variation of the $\mathrm{CH}-$ band intensity with temperature up to $600^{\circ} \mathrm{C}$ could be detected spectroscopically. The use of plasma simulators and edge plasmas in tokamaks has widely increased the data basis and measured chemical erosion yield data are available from the plasma simulators PSI-1 in Berlin and PISCES B in San Diego, and from plasma edge and divertor measurements in the fusion facilities JET, Tore Supra, TEXTOR, ASDEX Upgrade and JT-60U. However, until 1998 [1, 10] the flux dependence at high fluxes could not be clarified within the scatter of the available data.

Therefore, an attempt was made to normalize all data to the same conditions, such as particle energy and surface temperature. As from ion beams and plasma simulators most of the data were obtained at $30 \mathrm{eV}$, and all data from tokamaks were subsequently normalized to these conditions. All the authors have responded, and the data have indeed been measured at different ion energies and surface temperatures. For both quantities, sufficient information was obtained to 


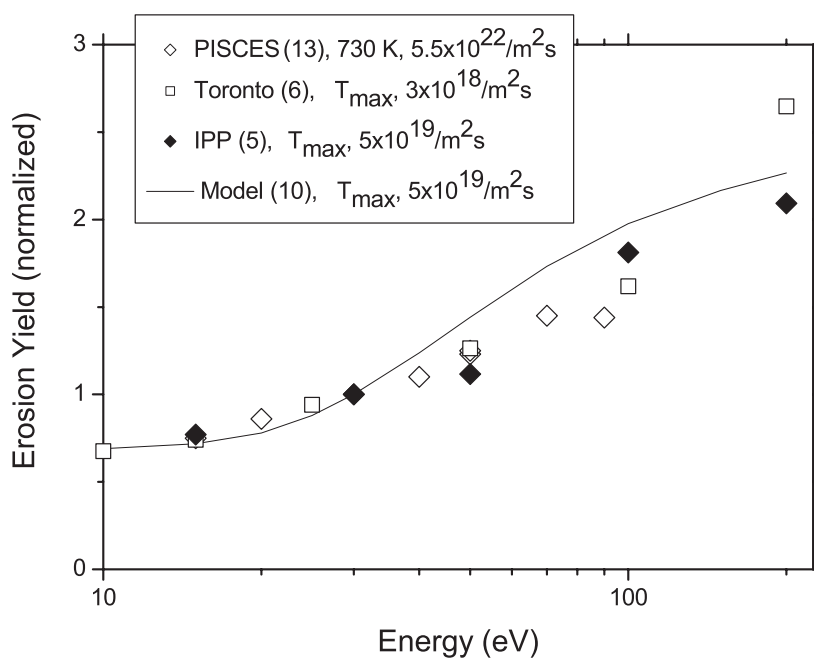

Figure 1. Energy dependence of chemical erosion as measured in ion beam studies [5,6] and PISCES-B [14] at the temperature of maximum yield, $T_{\max }$. The data are normalized at $30 \mathrm{eV}$ and fitted using the analytic model [10].

allow normalization to $30 \mathrm{eV}$ and select data from near the maximum chemical erosion, $T_{\max }$.

As a basis for the energy scaling, the energy dependence has been published for ion beams in $[4,10]$ or demonstrated in PISCES B [14] (figure 1). The temperature dependence was shown to be very broad at fluxes higher than $10^{22} \mathrm{~m}^{-2} \mathrm{~s}^{-1}$ (see [15] and figure 2), and data could be selected sufficiently close to $T_{\max }$, such that no normalization to $T_{\max }$ appears necessary between 600 and $1100 \mathrm{~K}$. This led to the following conclusions:

Ion beam data. As a low flux value, the ion beam data from IPP were taken. The determination of the total mass loss of the samples results in quantitative absolute erosion yield values including all emissions of saturated hydrocarbons and radicals. Data from residual gas analysis [5] may not fully account for hydrocarbon radicals with high surface sticking coefficients [6] and are considered only in their relative dependence on energy and temperature (figures 1 and 2). Data at $30 \mathrm{eV}$ were chosen at $T_{\max }$ [6]. Mass spectroscopic identification of hydrocarbon species was performed. Typically, in ion beam experiments the contribution of heavier hydrocarbons to the total erosion is about equal to that of methane (see [16]).

PSI-1 Berlin. The data set includes results published in [17] from the plasma generator PSI-1 and more recent data after upgrading from PSI-2 [18]. The ion flux density at the target position was exactly determined from the ion saturation current to a negatively biased Mo target. The erosion yield of the carbon materials was determined by optical spectroscopy of the $\mathrm{CD}$ band and by mass spectrometry. Both diagnostics were absolutely calibrated by injection of $\mathrm{CD}_{4}$ into the plasma near the target [19]. In [17] weight loss measurements were also performed. After measurements at fluxes close to $2 \times 10^{23} \mathrm{~m}^{-2} \mathrm{~s}^{-1}$ as published in [18], no further data were produced. The data were all measured at $T_{\max }$ and at an ion energy of $30 \mathrm{eV}$. The data were included in figure 4 without further normalization.

PISCES B. Also, the PISCES B data have not been changed since their publication in [14]. They were obtained

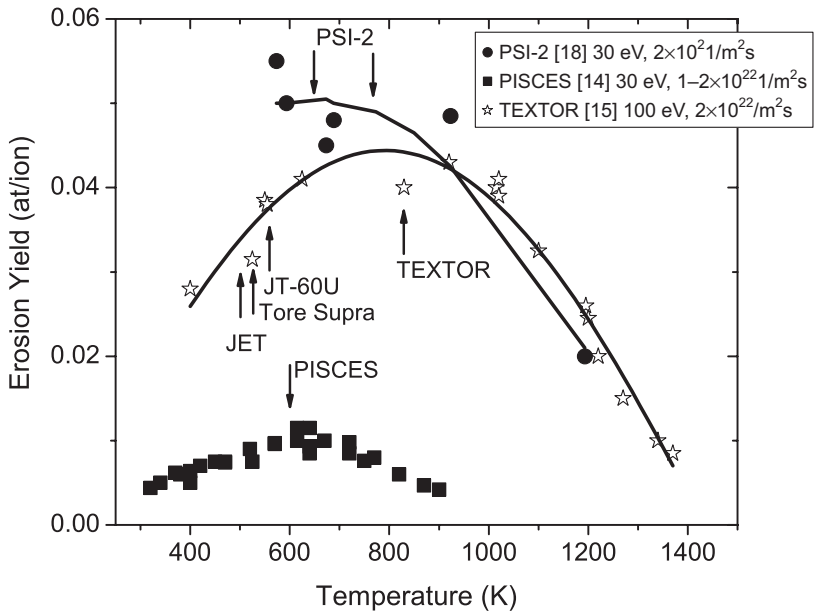

Figure 2. Temperature dependence of chemical erosion as measured in different high-flux devices. The large difference in absolute yield reflects the different conditions, i.e. ion flux and particle energy. The temperatures where the flux dependence of the chemical erosion yield was determined (see figure 4) are indicated for each device.

at a bias of $-30 \mathrm{~V}$, normalized to $T_{\max }$ using the measured temperature dependence (see figure 2). The optical spectroscopy of the CD band was calibrated with hydrocarbon gas puffs. The weight loss measurements had to be interpreted using a re-deposition code to obtain gross erosion yields. The published data were taken unchanged.

JET. In discussions with the authors it was concluded that the most reliable data are the ones that were taken from $\mathrm{CD}$ band measurements together with calibration by hydrocarbon gas puffs and published in [20]. The incident flux was determined from $\mathrm{D}_{\alpha}$ measurements using $S / X B=20$ and from Langmuir probe signals. As the JET data integrate over the whole outer divertor plasma, they contain contributions from a wide distribution of fluxes and energies. Stamp [21] provided the most significant flux values for the data points. For the energy no normalization could be done due to the integral nature of the data, the peak electron temperature varying between $20 \mathrm{eV}$ at low densities and $8 \mathrm{eV}$ at high densities. Within the restricted flux range of $5 \times 10^{22}$ $1.5 \times 10^{23} \mathrm{~m}^{-2} \mathrm{~s}^{-1}$ no obvious flux dependence can be observed for $\mathrm{CD}_{4}$ and $\mathrm{CH}_{4}$, the yield for heavier hydrocarbons being about equal to the methane yield.

Tore Supra. Two sets of data [22-24] are shown in figure 4, both measured in the pump duct by mass spectrometry of hydrocarbons produced at the neutralizer plate of the pumped limiter. The mass spectroscopic signal was calibrated by hydrocarbon gas puffs. The new data [23,24] showed values about a factor of 5 larger than the first set of data [22]. However, it was realized that the ionization probability of hydrocarbons in the pump duct leads to a loss of hydrocarbons to the walls before reaching the mass spectrometer. This had not been taken into account in the earlier data, although this factor was already estimated in the original publication to be about a factor of 7. After inclusion of this factor the discrepancy between the two sets of data disappeared. For all data points the plasma electron temperature was provided (between 9 and $25 \mathrm{eV}$ ) and normalization to an incident particle energy of $30 \mathrm{eV}$ could be done. The Tore Supra data for $\mathrm{CD}_{4}$ may represent a lower 
limit as in the later publication an increase of the yield data with increasing surface temperature from 250 to $550^{\circ} \mathrm{C}$ was found. As for JET, the contribution of heavier hydrocarbons was determined to be about equal to that of methane.

TEXTOR. The data shown in figure 4 were obtained spectroscopically at the TEXTOR test limiter and published in [15]. Both, density and limiter position scans were made and spectroscopic parameters were calibrated in situ by hydrocarbon gas puffs. A scan of radial positions of the test limiter leads to higher plasma temperatures for higher ion fluxes, in contrast to most other data measured under divertor conditions, where the plasma density was changed. For all data points the edge plasma temperature and density were provided. Actually, the TEXTOR data require the largest normalization factor as edge plasma temperatures in few cases reaching values of up to $60 \mathrm{eV}$. The energy normalization at the highest fluxes results in a factor of 0.5 , in good agreement with the energy scaling in the PISCES B experiment (see figure 1). As seen in JET and Tore Supra, heavier hydrocarbons contribute to the erosion yield about as much as methane does.

Recent experiments clarifying the flux dependence under different limiter geometries are presently under way in TEXTOR. Additionally, it seems that the hydrogen flux derived from the D-gamma line emission may have been the lower limit because of the molecular influence on the emission of the atomic line radiation. The hydrocarbon production yields should, therefore, be treated as the upper limit.

$J T-60 U$. The results for JT-60U shown in figure 4 were taken from the optical spectroscopic determination of the $\mathrm{CD}$ - and $\mathrm{C}_{2}$-band measurements at the outer divertor [25]. For all data points the plasma temperatures were provided, ranging from 7 to $19 \mathrm{eV}$, and normalized to the particle energy of $30 \mathrm{eV}$. Earlier data [26] from the inner divertor may have included detachment effects at the divertor plate with much lower plasma temperatures and are not included here.

The JT-60U data are the only ones not calibrated in situ by hydrocarbon gas puffs and rely on spectroscopic detection efficiencies $(D / X B)$ measured in PISCES [27]. The evaluation of spectroscopic signals for heavier hydrocarbons using the parameters from PISCES led to unusually high contributions of $\mathrm{C}_{2} \mathrm{D}_{x}$ hydrocarbons, up to a factor of 3.5 larger than that of methane. The reason for this strong contribution is not known and more investigation is required, if possible using in situ quantification by calibrated gas puffing. The $\mathrm{CD}_{4}$ data were considered for the resulting fit to the flux dependence as all other data, although the result from this joint endeavour is that the $D / X B$ must be in situ calibrated. JT-60U has finished installation of the gas inlet for the in situ calibration by using hydrocarbon gases and will be able to give revised sputtering yield data by the end of 2004 .

ASDEX Upgrade. ASDEX Upgrade is the only device in which the chemical erosion yield was measured at room temperature. As the erosion mechanism appears to be different from the thermal emission at elevated temperatures [3], no scaling of these data was done to $T_{\max }$. In ASDEX Upgrade the recent in situ calibration results in a slight dependence of the spectroscopic parameter, $D / X B$, on ion flux (i.e. electron density), while earlier evaluations [28] assume a constant $D / X B=50$. This measured dependence tends to soften

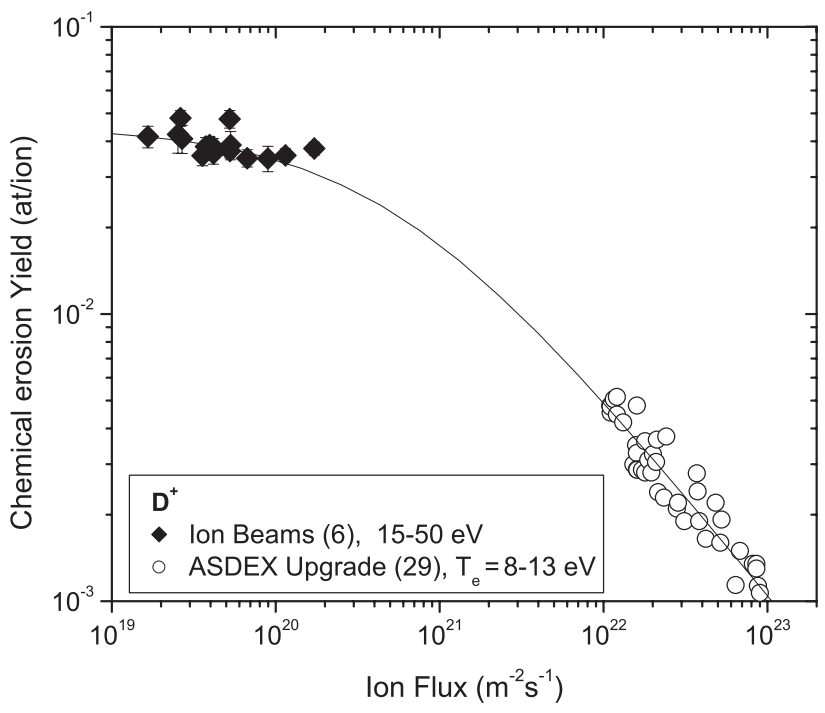

Figure 3. Flux dependence of the chemical erosion yield at room temperature, as measured in ion beam experiments [6] and in the divertor of ASDEX Upgrade [29]. The solid line is a fit to the data using equation (1), resulting in a flux dependence as $\Phi^{-0.7}$ at high fluxes.

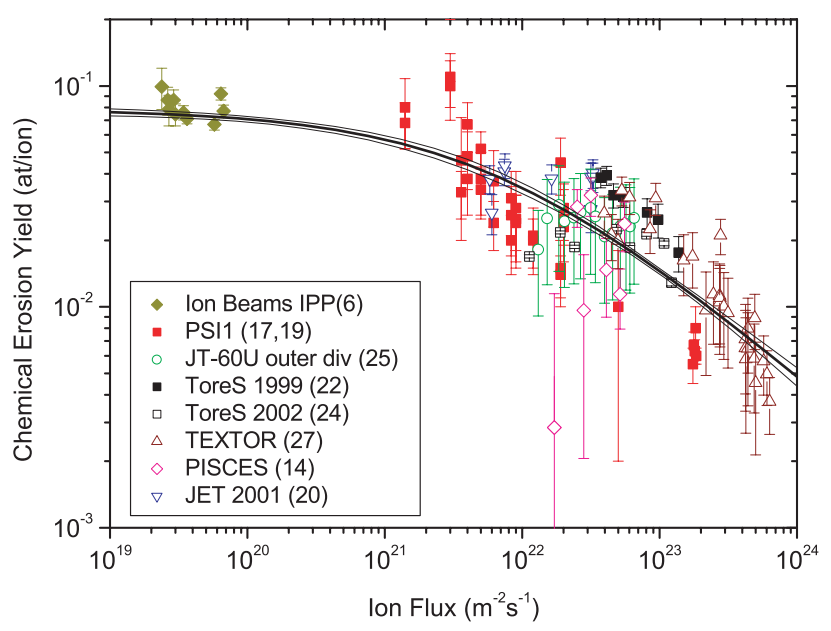

Figure 4. Flux dependence of the chemical erosion yield for $T_{\max }$ and an ion energy of $30 \mathrm{eV}$ determined from spectroscopic measurements in different fusion devices and plasma simulators. The solid lines are fits using Bayesian probability theory and its confidence intervals [31].

the decrease of the erosion yield with ion flux to values of about $\Phi^{-0.7}$ (see figure 3). All the ASDEX Upgrade measurements up to now have been performed considering only $\mathrm{D} / \mathrm{XB}$ of $\mathrm{CD}$ from $\mathrm{CD}_{4}$, and do not take into account directly higher hydrocarbons in the eroded flux and the effect of higher hydrocarbons on $D / X B$ for $C D$ from $\mathrm{CD}_{4}$. A detailed evaluation of the results is in progress $[29,30]$.

After this re-evaluation and normalization of the data, the following set of high-flux data for methane production at $T_{\max }$ is available (see figure 4). The data are for D ions, normalized to an incident ion energy of $30 \mathrm{eV}$ and considered to be taken at or near $T_{\max }$. While individual data sets in a narrow range of fluxes cannot distinguish clearly flux dependences (PISCES, JET, JT-60U) the ensemble of data points and the 
individual investigations of PSI-1, TEXTOR and Tore Supra, spanning flux ranges of more than an order of magnitude, suggest a decrease in the erosion yield with ion flux, $\Phi$, starting at fluxes of about $10^{21} \mathrm{~m}^{-2} \mathrm{~s}^{-1}$. All investigators had to provide error bars for their yield values in order to weigh the data according to their uncertainty level. The model for the functional dependence was

$$
Y=\frac{Y_{\text {low }}}{1+\left(\Phi / \Phi_{0}\right)^{\varepsilon}}
$$

where $Y_{\text {low }}$ is given by low-flux data from ion beams to 0.08 , $\Phi$ is the flux where the transition to a flux dependence occurs and $\varepsilon$ is the power of the decrease at high fluxes. A powerful and consistent method of fitting data of different sources and error bars is Bayesian probability theory, which was employed in order to determine the free parameters of the model function [31]. In this self-consistent framework, the results for the parameters are given as expectation values over posterior probability distributions of the data. Error margins of the result are automatically provided. In the limited space of this letter, more details on the application of the method for evaluating the flux dependence of chemical erosion cannot be given. For those details the readers are referred to [32]. The resulting fit indicates a decrease in the yield at high fluxes with $\varepsilon=0.54$. The thin solid lines in the figure give the confidence interval of the resulting fit, predicting a yield of $(5 \pm 0.5) \times 10^{-3}$ at a flux of $10^{24} \mathrm{~m}^{2} \mathrm{~s}^{-1}$, in contrast to previous conservative estimates of $3 \times 10^{-2}$ [10]. The fit to the data using Bayesian probability theory [32] resulted in a decrease in the yield at high fluxes according to

$$
Y(E, T, \Phi)=\frac{Y_{\text {low }}(E, T)}{1+\left(\Phi /\left(6 \times 10^{21}\right)\right)^{0.54}} .
$$

The flux dependence, as given in equation (2) for the erosion yield at $T_{\max }$, seems also to apply at room temperature (see figure 3). Therefore, it can be assumed that at all temperatures the same factor can be applied to the analytical expression for the chemical erosion yield as given previously [10]. With this flux dependence, a description is now available that covers the energy, temperature and flux dependence adequately for extrapolation to wall and divertor conditions in ITER. Preliminary estimates of the ITER divertor erosion and re-deposition indicate a reduction in the net erosion by about one order of magnitude, mostly due to the flux and temperature dependence, as compared with previous estimates using a constant erosion yield of $1.5 \%$. In the main erosion zone, occurring at the outer divertor plate, chemical erosion is reduced to values below physical erosion, while it still dominates at the inner divertor plate. Like the erosion, the co-deposition of $T$ will also be greatly reduced [33].

There still remain uncertainties in the detailed determination of the contribution of heavier hydrocarbons to the total erosion yield. In [10] an additional factor of 1.3 takes account of heavier hydrocarbons, but this factor needs further investigation, especially at low ion energies.

\section{References}

[1] Roth J. 1993 Atomic and Plasma-Material Interaction Processes in Controlled Thermonuclear Fusion ed R.K. Janev and H.W. Drawin (Amsterdam: Elsevier)

[2] Horn A., Schenk A., Biener J., Winter B., Lutterloh C., Wittmann M. and Küppers J. 1994 Chem. Phys. Lett. 231193

[3] Roth J. and García-Rosales C. 1996 Nucl. Fusion 361647 Roth J. and García-Rosales C. 1997 Nucl. Fusion 37897 (corrigendum)

[4] Mech B.V., Haasz A.A. and Davis J.W. 1998 J. Nucl. Mater. 255153

[5] Mech B.V., Haasz A.A. and Davis J.W. 1998 J. Appl. Phys. 841655

[6] Balden M. and Roth J. 2000 J. Nucl. Mater. 28039

[7] Wittmann M. and Küppers J. 1996 J. Nucl. Mater. 227186

[8] Vietzke E., Flaskamp K., Philipps V., Esser G., Wienhold P. and Winter J. 1987 J. Nucl. Mater. 145-147 443

[9] Yamada R., Nakamura K., Sone K. and Saidoh M. 1980 J. Nucl. Mater. 95278

[10] Roth J. 1999 J. Nucl. Mater. 266-269 51

[11] Roth J., Bohdansky J. and Wilson K.L. 1982 J. Nucl. Mater. 111-112 775

[12] Roth J. 1983 Sputtering by Particle Bombardment, II ed R. Behrisch (Berlin: Springer)

[13] Pitcher C.S. et al 1986 Nucl. Fusion 261641

[14] Whyte D.G., Tynan G.R., Doerner R.P. and Brooks J.N. 2001 Nucl. Fusion 4147

[15] Pospieszczyk A. 1999 Phys. Scr. T 8148

[16] Vietzke E. and Haasz A.A. 1996 Chemical erosion Physical Processes of the Interaction of Fusion Plasmas with Solids ed W. Hofer and J. Roth (San Diego: Academic) p 135

[17] Grote H. et al 1999 J. Nucl. Mater. 266-269 1059

[18] Kornejew P., Bohmeyer W., Reiner H.-D. and Wu C.H. 2001 Phys. Scr. T 9129

[19] Kornejew P., Bohmeyer W. and Reiner H.-D. 1990 Phys. Scr. T 8140

[20] Stamp M.F., Erents S.K., Fundamenski W., Matthews G.F. and Monk R.D. 2001 Phys. Scr. T 9113

[21] Stamp M.F. 2003 EURATOM/UKAEA Fusion Association, Culham Science Centre, Abingdon, UK, private communication

[22] Ruggieri R. et al 1999 J. Nucl. Mater. 266-269 660

[23] Cambe A., Gauthier E., Hogan J. and Layet J.M. 2002 Chemical erosion measurements in Tore Supra Meeting on Erosion, Deposition and Related Modelling Activities (Culham, 20-21 March 2002) at press

[24] Cambe A., Gauthier E., Hogan J. and Layet J.M. 2003 J. Nucl. Mater. 313-316 364

[25] Nakano T. et al 2002 Nucl. Fusion 42689

[26] Higashijima S. et al 1997 J. Nucl. Mater. 241-243 574

[27] Pospieszczyk A. et al 1989 UCLA-PPG-1251

[28] Kallenbach A. et al 1999 J. Nucl. Mater. 266-269 343

[29] Pugno R., Kirschner A., Krieger K., Kallenbach A. and Coster D. 2004 Last results form the hydrocarbon puff experiment in the ASEDX Upgrade divertor EU Task Force on PWI Meeting (Garching, February 2004) http://www.efda-taskforce-pwi.org/

[30] Pugno R. et al ASDEX Upgrade Team, Parameter dependence of carbon chemical erosion in ASDEX Upgrade divertor IIb, 16th PSI, Portland, Maine, J. Nucl. Mater. at press

[31] Dose V. 2003 Bayesian inference in physics: case studies Rep. Prog. Phys. 661421

[32] Dose V., Preuss R. and Roth J. 2001 J. Nucl. Mater. 288153

[33] Roth J. et al Flux dependence of chemical erosion and its implication for ITER 16th PSI, Portland, Maine, J. Nucl. Mater. submitted 